\title{
Pulse Excitation of Micro-Fluxgate Sensors
}

\author{
Pavel Ripka, San On Choi, Alois Tipek, Shoji Kawahito, and Makoto Ishida
}

\begin{abstract}
Miniature fluxgate sensors with symmetrical closed core elements on both sides of the planar coils were manufactured using standard microtechnology. The new sensors have shown substantial improvement over the standard single-sided microfluxgate sensors: for the same field range the sensor noise was reduced 10-times to $20 \mathrm{nT}$ rms $(20 \mathrm{mHz} \ldots 10 \mathrm{~Hz})$ and the perming suppressed below $5 \mu \mathrm{T}$, for field shocks of $6 \mathrm{mT}$. The maximum sensitivity for sinewave excitation was $32 \mathrm{~V} / \mu \mathrm{T}$ for $1 \mathrm{MHz}$ frequency and $200 \mathrm{~mA} p$ - $p$ excitation current amplitude. Pulse shape of the excitation current allows use of high current peaks to suppress perming, while the rms value is low. Using a $20 \%$ duty factor squarewave excitation with $180 \mathrm{~mA} p$ - $p$ amplitude, the sensitivity was twice that of the sinewave excitation, while the chip temperature dropped from $80^{\circ} \mathrm{C}$ to $40^{\circ} \mathrm{C}$.
\end{abstract}

Index Terms-Fluxgate, magnetic field sensors, magnetometers, microfluxgate.

\section{INTRODUCTION}

$\mathbf{T}$ RADITIONAL fluxgate sensors are popular for measuring the magnetic field in the range of $1 \mathrm{nT}$ to $1 \mathrm{mT}$. They can reach better than a $0.1 \mathrm{nT}$ resolution and high precision such as $10 \mathrm{ppm}$ linearity error and $30 \mathrm{ppm} /{ }^{\circ} \mathrm{C}$ temperature coefficient of sensitivity. These devices need to be manually adjusted and individually calibrated which causes manufacturability issues, and leads to expensive devices [1], [2].

Many applications require cheap sensors or sensor arrays with a $10 \mathrm{nT}$ to $1 \mathrm{nT}$ resolution. These include magnetic ink reading, detection of ferromagnetic objects such as weapons and vehicles, reading of magnetic labels, magnetic 3-dimensional position tracking for virtual reality systems and robots [3]. Some of these applications can be successfully addressed by ferromagnetic magnetoresistors: commercially available AMR with flipping and newly developed linear GMR with a AC bias [4]. But there is still a strong demand for development of cheap and small vectorial magnetic field sensors, which could offer better accuracy than magnetoresistors.

Microelectronic technology has already been used to lower the production cost and further decrease the size of the fluxgate sensors. The first approach is to replace the excitation and

Manuscript received October 13, 2000.

This work was supported in part by the Czech Ministery of Education under Grant no. ME 275.

P. Ripka is with the Czech Technical University of Measurement, Fac. of Electr. Eng. CTU, Czech Republic (e-mail: ripka@ feld.cvut.cz).

S. O. Choi is with Samsung Advanced Institute of Technology, Suwon, Korea, 440-600.

A. Tipek is with the Czech Technical University of Measurement, Fac. of Electr. Eng. CTU, Czech Republic (e-mail: xtipeka@ @eld.cvut.cz).

S. Kawahito is with Research Institute of Electronics, Shizuoka University, 3-5-1, Johoku, Hamamatsu, 432-8011, Japan (e-mail: kawahito@idl.rie.shizuoka.ac.jp).

M. Ishida is with Dept. Electrical and Electronic Eng. Toyohashi Univ. of

Technol., Tempaku-cho, Toyohashi, Japan (e-mail: ishida@eee.tut.ac.jp).

Publisher Item Identifier S 0018-9464(01)06198-2.

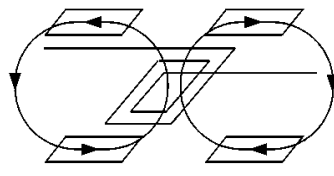

a)
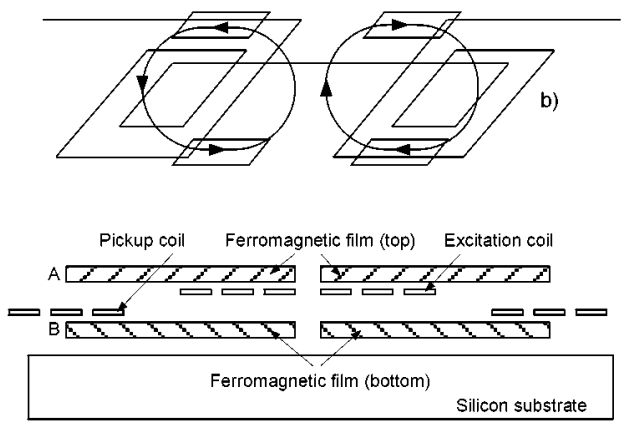

c)

Fig. 1. Double-sided microfluxgate sensor: (a) excitation coil and the flux path (pick-up coils are not shown for the clarity), (b) function of the pick-up coils, (c) cross-sectional view of the complete sensor.

sensing wire coils by solenoids made by pcb-technology [5], micromachining [6], or standard planar process [7], [8]. This geometry is ideal for the sensor function; problem is the manufacturing complexity and a limited number of turns of such solenoids. Another approach, also used in the present paper, is to use flat coils made by the planar process [9], [10].

\section{SENSOR DESIGN}

The new type of miniature fluxgate sensors with a symmetrical closed core and planar coils was manufactured using standard microtechnology [11]. The sensor geometry is shown in Fig. 1. The structure consists of two metallic layers made of $3 \mu \mathrm{m}$ aluminum, which are sandwiched between two ferromagnetic layers. The metallic layers form a flat excitation coil and two antiserially connected sensing coils. Four $0.7 \mathrm{~mm}$ long, $0.4 \mu \mathrm{m}$ thick core strips made of electroplated permalloy are positioned symmetrically on the both sides of the coils, so that they form two closed magnetic paths for the excitation field. Fig. 1(a) shows the centrally positioned excitation coil with two symmetrical excitation flux paths. The sensing coils are shown separaTely for clarity in Fig. 1(b). The cross-section of the complete sensor is shown in Fig. 1(c). The sensor is functionally similar to two double-core fluxgate sensors. The gain of this new design is that the sensor core has a high apparent permeability with respect to the excitation field so that the core may be deeply saturated to erase perming and reduce hysteresis. The apparent permeability with respect to the measured field is much lower, which results in higher sensor stability in the open-loop mode: temperature changes of the core material permeability 
practically does not influence the apparent permeability, which is mainly given by the core geometry.

The technical details of the new sensor are:

Chip size: $\quad 2.5 \mathrm{~mm} \times 4 \mathrm{~mm}$

Core material: $\mathrm{NiFe}$ (electroplated)

Core thickness: $4 \mu \mathrm{m}$

Core size: $\quad 700 \mu \mathrm{m} \times 1 \mathrm{~mm} \times 2$

Coil pitch: $\quad 15 \mu \mathrm{m}$ width $/ 5 \mu \mathrm{m}$ space $\rightarrow 20 \mu \mathrm{m}$ pitch

Coil thickness: $3 \mu \mathrm{m}$

Coil turns: $\quad 40$ each for pickup and excitation

\section{EXCITATION}

Micro-fluxgate sensors should be excited at a high frequency in order to increase their sensitivity, which is low as the number of turns of the sensing coil is limited. The sensor parameters for sinewave excitation were described in [11], [12]. Maximum sensitivity of $32 \mathrm{~V} / \mathrm{T}$ was reached for $1 \mathrm{MHz}, 220 \mathrm{~mA} p$ - $p$ excitation. The linear range in the open-loop was $1 \mathrm{mT}$, the hysteresis was $2.5 \mu \mathrm{T}$ (compared to $30 \mu \mathrm{T}$ for the single-core sensor) and also perming was reduced from $50 \mu \mathrm{T}$ to $5 \mu \mathrm{T}$ (for $6 \mathrm{mT}$ field shock in any direction).

The main drawback of the new sensor is the high resistance of the excitation coil, which is given by the technology used. When excited by a high amplitude sinewave, the sensor chip is excessively heated which results in nonstability of its characteristics and also in limited reliability.

Use of short pulses for excitation allows us to decrease the current rms level and thus the heating, while the current amplitude can even be increased, which was shown to reduce noise and further suppress the perming. Using a $20 \%$ duty factor squarewave excitation and $180 \mathrm{~mA} p$-p , the sensitivity was twice that of the sinewave excitation, while the chip temperature dropped from $80^{\circ} \mathrm{C}$ to $40^{\circ} \mathrm{C}$. Symmetrical driving circuits and good impedance matching was necessary to prevent reflected waves and distortion of fast signals caused by the parasitic capacitances.

The principle diagram of the excitation power stage is shown in Fig. 2. The bridge made of low on-resistance and fast Hexfet transistors is controlled by two squarewaves in anti-phase. Supplying the sensors through normal shielded cable brings problems with reflected waves and ringing. This can be solved either by placing the power stage into the sensor chip, or by using a fully balanced connection by two coaxial cables and terminal resistors. The correct current waveform for sensor supplied by a $1.5 \mathrm{~m}$ long cable is shown in Fig. 3(a). Fig. 3(b) shows the sensor output for a zero measured field. The ringing at high frequencies caused by parasitic capacitances is easily filtered off by processing circuits.

\section{MeAsured Values AND Discussion}

The double-side sensor properties for excitation current in the form of short pulses with variable duty factor, amplitude and repetitive frequency were measured. Here are presented only the results for the optimum repetitive frequency of $500 \mathrm{kHz}$.

The sensor output was measured by SR 844 RF lock-in amplifier SR 844 with a reference adjusted to $1 \mathrm{MHz}$ (2nd harmonics).

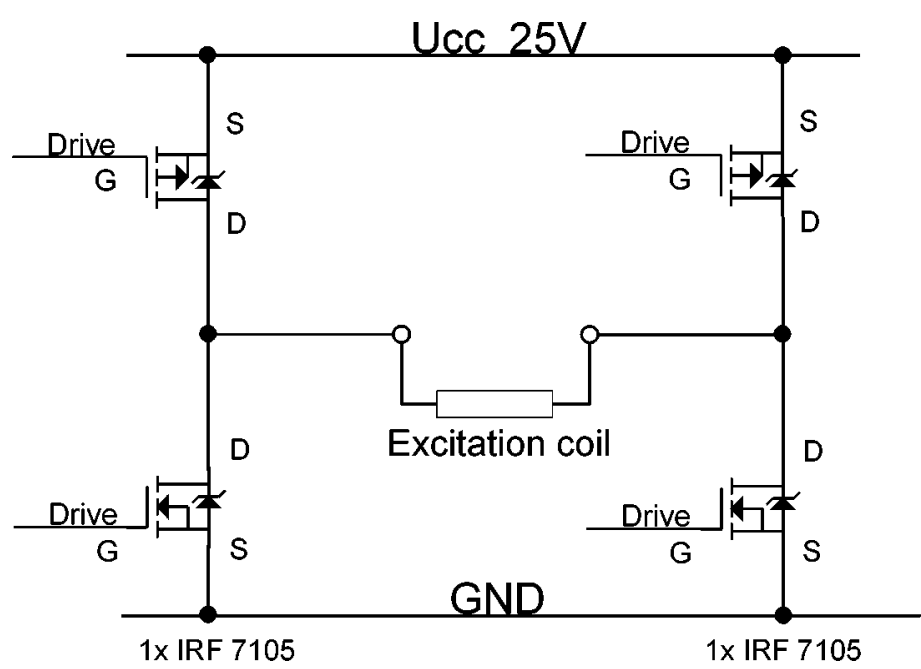

Fig. 2. Excitation circuit.

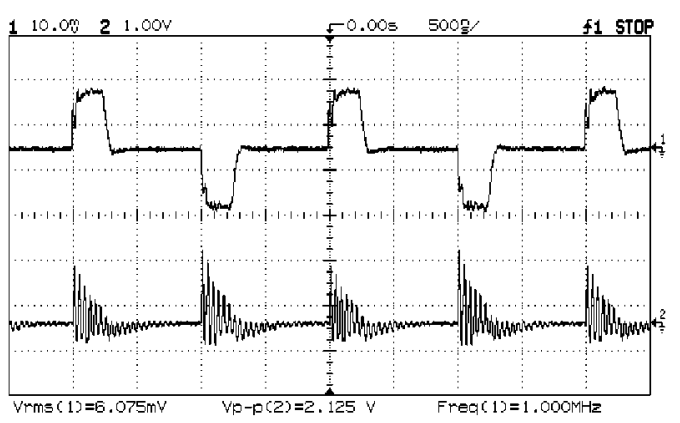

Fig. 3. Waveforms of the pulse-excited micro-fluxgate sensor: (a) Excitation current waveform of the sensor supplied through $1.5 \mathrm{~m}$ of symmetrical double coaxial cable and properly terminated $(100 \mathrm{~mA} / \mathrm{div})(\mathrm{b})$ output voltage at the pick-up coil (1 V/div) for zero measured field. The time scale is $500 \mathrm{~ns} / \mathrm{div}$.

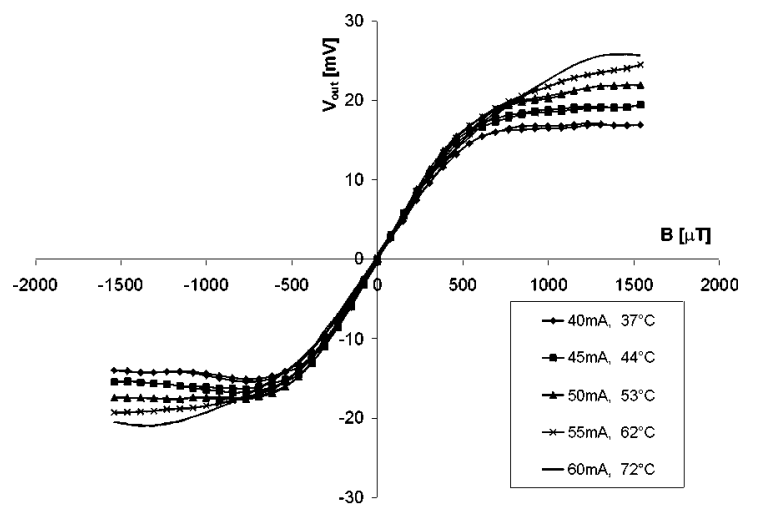

Fig. 4. Large-field characteristics of the micro-fluxgate excited by $500 \mathrm{kHz}$, $20 \%$ duty factor squarewave. Rms value of the excitation current is a parameter.

This instrument has a switching-type detector, so it is sensitive also to odd harmonics of the reference, i.e., to 6 th, 10 th, ... harmonics of the excitation frequency.

Fig. 4 shows the high-field characteristics of the sensor supplied by a $20 \%$ duty-factor squarewave for several values of the excitation current rms value. The linear range was $0.5 \mathrm{mT}$ which is a surprisingly lower value then for sinewave excitation. The sensor sensitivity and chip temperature as a function of the excitation current rms value are shown in Fig. 5. $55 \mathrm{~mA}$ rms excitation was selected as an optimum. Fig. 6 shows the sensor 


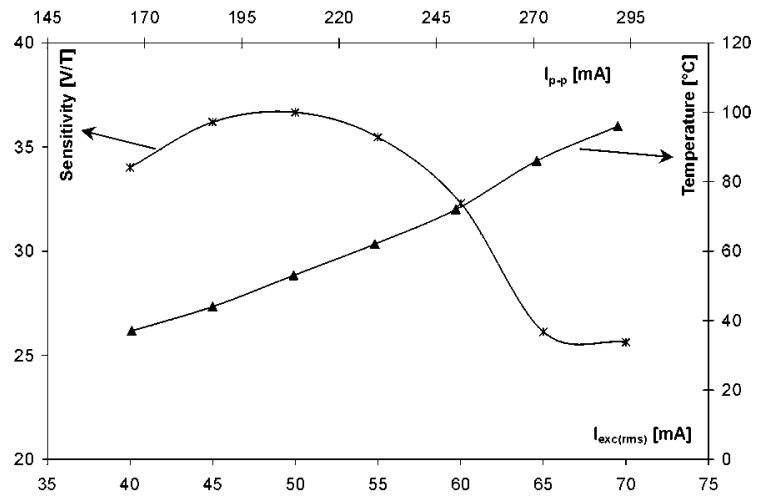

Fig. 5. Sensitivity and chip temperature as a function of rms value of the excitation current $(500 \mathrm{kHz}, 20 \%$ duty factor squarewave).

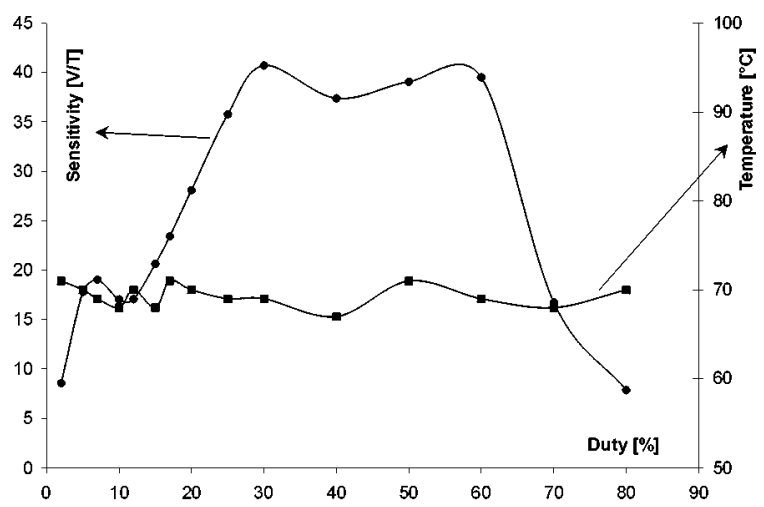

Fig. 6. Sensitivity and chip temperature as a function of the duty factor (500 $\mathrm{kHz}, 55 \mathrm{~mA}$ rms squarewave).

performance for this value as a function of the pulse duty factor. Except for very short pulses, chip temperature is given by the current rms value. The sensitivity is flat around $10 \%$ and $50 \%$ duty factors. Although the sensitivity is lower, the small duty factor is preferable as it allows a much higher core saturation. If we permit a chip temperature of $55^{\circ} \mathrm{C}$, the allowable rms excitation current is $55 \mathrm{~mA}$. The corresponding sinewave $p-p$ current is $156 \mathrm{~mA}$ for the sinewave, $230 \mathrm{~mA}$ for $20 \%$ duty-factor squarewave and $920 \mathrm{~mA}$ for the minimum allowable duty factor of 5 .

The measured noise level was $20 \mathrm{nT}$ rms $(20 \mathrm{mHz} \ldots 10 \mathrm{~Hz})$, i.e., it was was 10-times reduced with respect to single-sided core sensor. The perming was below $5 \mu \mathrm{T}$ (for field shocks of $6 \mathrm{mT})$.

\section{CONCLUSION}

Pulse excitation improves the properties of the micro-fluxgate sensors. Even the sensors manufactured in low-cost technology, with a thin metallic layer can be excited by strong peaks, which reduce the perming and noise. The sensor sensitivity can be increased by lowering the thickness of the permalloy layer, which would simultaneously reduce the sensor range.

The presented microfluxgate sensor works in the open loop, which limits the achievable linearity to about $1 \%$, still enough for many monitoring and industrial applications. In general, the advantage of using feedback in flat-coil sensors is questionable, as the compensation field is nonhomogenous.

Pulse-excited $2.5 \mathrm{~mm} \times 4 \mathrm{~mm}$ microfluxgate sensor with double layer permalloy has $20 \mathrm{nT}$ rms noise, $2.5 \mu \mathrm{T}$ hysteresis in $1 \mathrm{mT}$ range and $5 \mu \mathrm{T}$ perming error for $6 \mathrm{mT}$ field shock. The power consumption can be lowered by increasing the thickness of the metallic layer.

\section{REFERENCES}

[1] O. V. Nielsen et al., "Development, construction and analysis of the 'Orsted' fluxgate magnetometer," Meas. Sci. Technol., vol. 6, pp. 1099-1115, 1995.

[2] P. Ripka, "New directions in fluxgate sensors," JMMM, vol. 215-216, pp. 735-739, 2000.

[3] P. Ripka, Ed., Magnetic Sensors and Magnetometers. Boston, MA, London: Artech, 2001.

[4] P. Ripka, M. Tondra, J. Stokes, and R. Beech, "AC-Driven AMR and GMR magnetoresistors," Sensors and Actuators A, vol. 76, pp. 227-232, 1999 .

[5] O. Dezuari, E. Belloy, S. E. Gilbert, and M. A. M. Gijs, "Printed circuit board integrated fluxgate sensor," Sensors and Actuators A, vol. 81, pp. 200-203, 2000.

[6] T. M. Liakopoulos and C. H. Ahn, "A micro-fluxgate magnetic sensor using micromachined planar solenoid coils," Sensors and Actuators A, vol. 77, pp. 66-72, 1999.

[7] R. Gottfried et al., "A miniaturized magnetic-field sensor system consisting of a planar fluxgate sensor and a CMOS readout circuity," Sensors and Actuators A, vol. 54, pp. 443-447, 1996.

[8] S. Kawahito, H. Satoh, M. Sutoh, and Y. Tadokoro, "High-resolution micro-fluxgate sensing elements using closely coupled coil structures," Sensors and Actuators A, vol. 54, pp. 612-617, 1996.

[9] P. Kejik et al., "A new compact 2D planar fluxgate sensor with amorphous metal core," Sensors and Actuators A, vol. 81, pp. 200-203, 2000.

[10] S. O. Choi, S. Kawahito, Y. Matsumoto, M. Ishida, and Y. Tadokoro, "An integrated micro fluxgate magnetic sensor," Sensors and Actuators, vol. 55, pp. 121-126, 1996.

[11] P. Ripka, S. O. Choi, S. Kawahito, A. Tipek, and M. Ishida, "Microfluxgate sensor with double-sided core," presented at the Proc. EMSA 2000, vol. 167 168, Dresden. subm. for Sensors and Actuators.

[12] P. Ripka, S. O. Choi, A. Tipek, S. Kawahito, and M. Ishida, "Symmetrical core improves micro-fluxgate sensors," in Proc. Eurosensors 2000, Copenhagen, pp. 499-500. 